\title{
Cell regeneration medicine in case of salih myopathy and hypoxic encephalopathy
}

Keywords: stem cell, bone marrow, adipose tissue cells, immunomodulatory effect

Abbreviations: CMM, stem cells; CMMGW, warthon gelatin stem cells; GW, warthon's jelly; HMCII, histocompatibility antigen type II; CD, marker molecules on the cell surface; WG: warthon's gelatin; BDNF, brain derived neurotrofic factor; IGF 1, insulin growth factor type 1; TRH, thyrotropin-releasing hormone; ACTH, adrenocortiotropic hormone; TGF-B, beta transformation Stimulating Factor

\section{Introduction}

Currently there are several sources from which to perform stem cell extraction (MSC), among the most relevant and with the most promising uses are bone marrow, adipose tissue cells and Warthon gelatin cells (CMMWG). The latter comes from the umbilical cord, tissue specialized in the production of stem cells with the characteristics of high capacity for self-renewal and multiple differentiation, and the great advantage that its use does not present ethical restrictions since it is disposed of after delivery. ${ }^{1,2}$ Stem cells obtained from (GW) have low immunogenicity due to the fact that they do not activate the proliferation of $\mathrm{T}$ cells, as they do not express the main histocompatibility complex class II (MHCII) or the cd40, cd80 and cd86 molecules, necessary for the activation of $t$ cells. ${ }^{3}$ The world literature has not shown that when CMMGW is administered, the proliferation of lymphocytes is suppressed and also does not activate mhc20. For this reason, rejection after cell graft transplantation is minimal or null, even among individuals without any consanguinity. ${ }^{4}$ Another beneficial effect is that MSCs have an immunomodulatory effect on $\mathrm{T}$ and B lymphocytes, dendritic cells and NK cells, since it can inhibit the proliferation of immune cells and reduce the secretion of cytokines and the presence of the subtypes of cells infiltrated in tissue., ${ }^{3,5-8}$ which attenuates inflammation and promotes wound healing. These characteristics and properties of allogeneic CMMGW provide an advantage for tissue regeneration and thus achieve wound closure and avoid rejection..$^{9-12}$

Among the orphan and rare diseases we find salih myopathy, a hereditary musculoskeletal pathology, which developed as a delay in motor skills such as the inability to get up, sit or walk, associated with contractures that restrict movement of the neck or back, and on some occasions generating dilated cardiomyopathies, thus preventing the maintenance of cardiac output and perfusion in the other organs. This autosomal recessive phenomenon is produced by an alteration in the TTN gene, located on the long arm of chromosome 2 (2q31); which codes for the Tinin protein, vital for the proper functioning of the sarcomere. ${ }^{13-19}$

\section{Clinical case}

1-year-old male patient with a diagnosis of Salih myopathy, hypoxic encephalopathy, cerebral palsy, gastroesophageal reflux. He entered the Cell Regeneration Medical Organization (January 2020), where he was assessed by an interdisciplinary team (Dr. Medicine of cell regeneration, Md Anesthesiologist, Md physiatrist) finding: alterations in alertness (stupor), temporal-spatial disorientation,
Volume 6 Issue 5 - 2020

\author{
Felipe Torres Obando,' J Manuel Velasco, ${ }^{2} \mathrm{~N}$ \\ Adriana Soto, ${ }^{3}$ D Alejandro Vergara ${ }^{4}$ \\ 'Medical and Cientific Director, Colombia \\ ${ }^{2}$ Director of Scientific Studies - Md Epidemiologo, Colombia \\ ${ }^{3}$ Cell Regeneration Medicine Research Team, Colombia \\ ${ }^{4}$ Cell Regeneration Medicine organization, Colombia
}

Correspondence: J Manuel Velasco, Director of Sientific Studies - Md Epidemiologo, Colombia, Email juan.velasco@crmedicine.com

Received: September 22, 2020 | Published: October 26, 2020

language, presents praxis or generalized involuntary movements, neurogenic bladder with evacuating catheterizations for 4 months, severe swallowing disorder, requires nutrition by gastrostomy, presents generalized spasticity, at cardiorespiratory level, rhythmic heart sounds there is no presence of murmurs and in the pulmonary auscultation there is the presence of scattered rhonchi in both lung fields. The diagnosis of Salih myopathy was made by complete PCR amplification of the exons of the TTN gene and their subsequent sequencing (an extra-institutional examination). Within the laboratory tests without alteration in coagulation times, without signs of infection, with a normal platelet leakage which would refer us to perform ultrasound implantations with minimal risk. For this reason, the medical board decided to endorse cell regeneration therapy with CMMGW combined with nanopharmacology, diamagnetotherapy, during its initial assessment it is applied to an instructional assessment tool, see annex 1 before the start of treatment, with the aim of having an examination with quantifiable nominal variables in order to show the progressive changes that occur during its post-treatment evolution. The patient is evaluated 3 and 6 months after treatment with the same tool.

\section{Regeneration therapy}

Cell therapy: In surgery rooms CLÍNICA INO Bogotá Colombia (aseptic environment, CMMGW implantations are performed with two specific objectives: immediate response implantations (intrathecal implantation, intravenous implantation), and depot implantation (ultrasound-guided cervical epidural implantation, implantation intramuscular) for a total of 100 million CMMGW combined with specific somatic tissue cells of the muscle, the diencephalon and the cerebral cortex in order to have better adaptations to the responses of your encephalopathy and myopathy.

Diamagnetic therapy: By means of the CTU mega 20 machine, diamagnetic therapy was performed for stimulation and migration of neuronal plasticity 7 sessions. 
Microbiological therapy: probiotics in high doses for a period of 4 months, with 6 different strains, together with orthomolecular nutrition (nutrients in dose cans) which allows us to stimulate defense systems and hormonal and endocrine regulation.

Physiological regulation: BDNF (brain derived neuotrofic factor), neurotrophin 3, neurotrophin 4, melatonin, insulin growth factor type 1 (IGF1), coenzyme Q10, ACTH oxytocin, TRH, b-endorphin, TGFB, anti-interleukin 1, Interleukin 10, Enzyme catalysts, RNA and DNA recombine.

Molecular activation: DEPREXIL, which stimulates neuronal development, and improves cellular oxidation at the central level, and CELL ORGAN helps muscle and neuronal development. PROCUMIN (doses of essential and non-essential amino acids in high doses for the stimulation of your brain and your muscles).

\section{Results}

6 months after cell implantation, and the establishment of all regeneration medicine treatment, the patient showed a significant evolution towards improvement. We found that their level of attention allows them to be more connected with the environment, fix and recognize objects better, spasticity persists but less with respect to the beginning, improvement in swallowing of saliva in no longer requiring bladder catheterizations for 3 months, improvement of their secretions and bronchial hyperresponsiveness, see annex 2 .

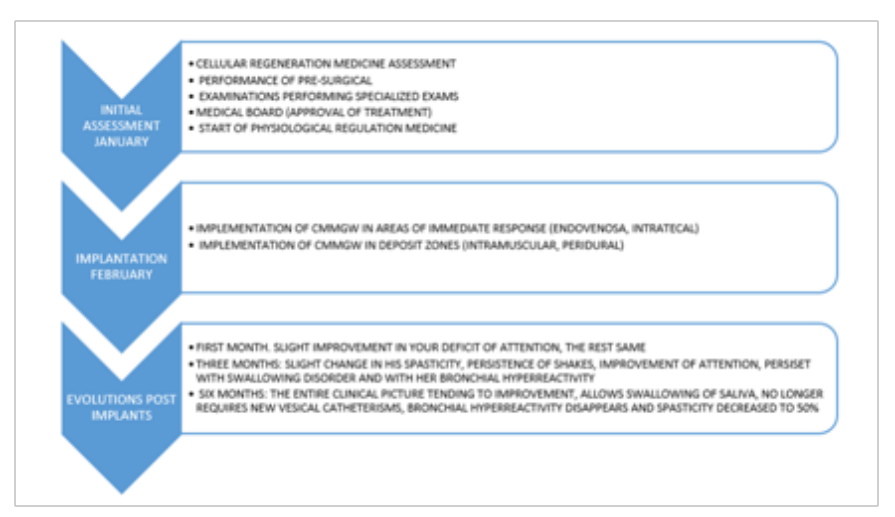

\section{Discussion}

Salih myopathy has a low incidence and prevalence worldwide. It is also known as early-onset myopathy with fatal heart disease, and until now there is no specific type of treatment known to control the symptoms or cure the disease.

It is categorized as orphan and rare, whose cases are rare worldwide. For this reason, finding a significant cohort to carry out a study that promotes this therapy is difficult; However, at Cell Regeneration medical Organization, a recognized international center for the treatment of orphan and rare diseases, we offered a treatment based on physiological regulation medicine and cell therapy with CMMGW obtained from the umbilical cord of his brother. It was decided to apply this type of cell therapy due to its low immunogenicity, thus allowing to minimize any type of rejection caused in implantation and it is combined with nanopharmacology, physiological regulation, microbiological therapy and diamagnetotherapy to modulate, activate and stimulate the cellular response directed at the population. target (central nervous system and striated muscular system). This incorporation of a combination of different technologies has allowed not only to achieve excellent results that go from the brake or arrest of the disease but also the region of the pathology to minor or initial stages.

The results obtained during the patient's evolution always showed a clear trend towards improvement, from the first month to 6 months after the implant. Achieving attenuation of some signs and repairing others as presented with the neurogenic bladder.

\section{Conclusion}

Physiological regeneration medicine treatment based on cell therapy (CMMGW) in conjunction with the implementation of different therapeutic tools (nanopharmacology, microbiological therapy and diamagnetotherapy), are considered a life expectancy for the patient and an interesting medical alternative in the fight for discover possible treatments that are more effective than the current ones not only for salih myopathy but also for other types of rare orphan diseases.

\section{Acknowledgments}

None.

\section{Conflicts of interest}

Author declare that there is no conflict of interest.

\section{Funding}

None.

\section{References}

1. Bongso A, Fong CY, Gauthaman K. Taking stem cells to the clinic: major challenges. J Cell Biochem. 2008;105:1352-1360.

2. Gao LR, Zhang NK, Ding QA, et al. Common expression of stemness molecular markers and early cardiac transcription factors in human Wharton's jelly-derived mesenchymal stem cells and embryonic stem cells. Cell Transplant. 2013;22(10):1883-1900.

3. Weiss ML, Anderson C, Medicetty S, et al. Immune properties of human umbilical cord Wharton's jelly-derived cells. Stem Cells. 2008;26:28652874.

4. Li X, Bai J, Ji X, et al. Comprehensive characterization of four different populations of human mesenchymal stem cells as regards their immune properties, proliferation and differentiation. Int $\mathrm{J} \mathrm{Mol} \mathrm{Med.}$ 2014;34(3):695-704.

5. Le Blanc K, Tammik L, Sundberg B, et al. Mesenchymal stem cells inhibit and stimulate mixed lymphocyte cultures and mitogenic responses independently of the major histocompatibility complex. Scand J Immunol. 2003;57:11-20.

6. Magatti M, De Munari S, Vertua E, et al. Human amnion mesenchyme harbors cells with allogeneic T-cell suppression and stimulation capabilities. Stem Cells. 2008;26:182-192.

7. Asari S, Itakura S, Ferreri K, et al. Mesenchymal stem cells suppress B-cell terminal differentiation. Exp Hematol. 2009;37:604-615.

8. Spaggiari GM, Capobianco A, Abdelrazik H, et al. Mesenchymal stem cells inhibit natural killer-cell proliferation, cytotoxicity, and cytokine production: role of indoleamine 2,3-dioxygenase and prostaglandin E2. Blood. 2008;111:1327-1333.

9. English K, Barry FP, Mahon BP. Murine mesenchymal stem cells suppress dendritic cell migration, maturation and antigen presentation. Immunol Lett. 2008;115:50-58.

10. Dominici M, Le Blanc K, Mueller I, et al. Minimal criteria for defining multipotent mesenchymal stromal cells. The International Society for Cellular Therapy position statement. Cytotherapy. 2006;8:315-317. 
11. Benítez-Arvizu G, Palma-Lara I, Vázquez-Campos R, et al. Autologous mesenchymal stem cells and cutaneus autograft as a treatment for chronic ulcer secondary to diabetes mellitus 2. Cir Cir. 2015;83:532-536.

12. Midwood KS, Williams LV, Schwarzbauer JE. Tissue repair and the dynamics of the extracellular matrix. Int $\mathrm{J}$ Biochem Cell Biol. 2004;36:1031-1037.

13. Sam S, Mazzone T. Adipose tissue changes in obesity and the impact on metabolic function. Transl Res. 2014;164:284-292.

14. Pittenger MF, Mackay AM, Beck SC, et al. Multilineage potential of adult human mesenchymal stem cells. Science. 1999;284:143-147.

15. Stadelmann WK, Digenis AG, Tobin GR. Physiology and healing dynamics of chronic cutaneous wounds. Am J Surg. 1998;176:26S-38S.
16. Iba Y, Shibata A, Kato M, et al. Possible involvement of mast cells in collagen remodeling in the late phase of cutaneous wound healing in mice. Int Immunopharmacol. 2004;4:1873-1880.

17. Chapel A, Bertho JM, Bensidhoum M, et al. Mesenchymal stem cells home to injured tissues when co-infused with hematopoietic cells to treat a radiation-induced multi-organ failure syndrome. $J$ Gene Med. 2003;5:1028-1038.

18. Maxson S, López EA, Yoo D, et al. Concise review: role of mesenchymal stem cells in wound repair. Stem Cells Transl Med. 2012;1(2):142-149.

19. Chang HY, Sneddon JB, Alizadeh AA, et al. Gene expression signature of fibroblast serum response predicts human cancer progression: similarities between tumors and wounds. PLoS Biol. 2004;2:E7. 